INPLASY



To cite: Guo. The effect of extraction on the upper airway in adult and adolescent patients: A systematic review and meta-analysis. Inplasy protocol 202040175. doi: 10.37766/inplasy2020.4.0175

Received: 25 April 2020

Published: 25 April 2020

Corresponding author: Runzhi Guo

runzhiguo2017@163.com

Author Affiliation:

Peking University

Support: None

Review Stage at time of this submission: Formal screening of search results against eligibility criteria.

Conflicts of interest: None to declare.

\section{The effect of extraction on the upper airway in adult and adolescent patients: A systematic review and meta-analysis}

Guo RZ.

Review question / Objective: The aim of this systematic review and meta-analysis was to analyze the effect of extraction on upper airway in adult and adolescent patients, and compare the difference between CBCT and cephalometric analysis in upper airway.

Condition being studied: The orthodontic treatment goal is not only the esthetic and stability, but also the function and health. One of the concerns orthodontists should be considered is the respiratory function, especially the upper airway. The upper airway consists of nasopharynx, oropharynx and hypopharynx. Among these, the oropharynx was surrounded by soft tissue (soft palate, tongue, and pharyngeal wall) without skeletal support, which could be easily affected. Changes in the upper airway dimension have been reported following the rapid maxillary expansion, the use of mandibular advancement appliance, and orthognathic surgery. Dental extractions are widely used to alleviate crowding, reduce facial convexity and correct anteroposterior discrepancies. Recently, the effect of extraction on the upper airway has gained increased interest from researchers. Despite a mount of clinical studies assessing the airway changes after extraction treatment, the effect of extraction on upper airway remains controversial.

INPLASY registration number: This protocol was registered with the International Platform of Registered Systematic Review and Meta-Analysis Protocols (INPLASY) on 25 April 2020 and was last updated on 25 April 2020 (registration number INPLASY202040175).

\section{INTRODUCTION}

Review question / Objective: The aim of this systematic review and meta-analysis was to analyze the effect of extraction on upper airway in adult and adolescent patients, and compare the difference between CBCT and cephalometric analysis in upper airway.

Condition being studied: The orthodontic treatment goal is not only the esthetic and 
stability, but also the function and health. One of the concerns orthodontists should be considered is the respiratory function, especially the upper airway. The upper airway consists of nasopharynx, oropharynx and hypopharynx. Among these, the oropharynx was surrounded by soft tissue (soft palate, tongue, and pharyngeal wall) without skeletal support, which could be easily affected. Changes in the upper airway dimension have been reported following the rapid maxillary expansion, the use of mandibular advancement appliance, and orthognathic surgery. Dental extractions are widely used to alleviate crowding, reduce facial convexity and correct anteroposterior discrepancies. Recently, the effect of extraction on the upper airway has gained increased interest from researchers. Despite a mount of clinical studies assessing the airway changes after extraction treatment, the effect of extraction on upper airway remains controversial.

\section{METHODS}

Participant or population: Orthodontic patients with premolars extracted were included without age or gender restriction.

Intervention: Orthodontic treatment with premolars extractions. The type of appliance, technique and treatment duration were not restricted.

Comparator: The untreated patients or orthodontic patients without extraction.

Study designs to be included: The randomized controlled trials (RCTs), controlled clinical trials (CCTs), cohort studies, and self-controlled studies were included.

Eligibility criteria: Longitudinal studies using CBCT or lateral cephalogram to observe the effect of extraction on upper airway were included.

Information sources: The electronic search of Embase, Cochrane Library, and Medline (via PubMed) databases. Manual screening of three major orthodontic journals (European Journal of Orthodontics, American Journal of Orthodontics and Dentofacial Orthopedics, and Angle Orthodontist), and the reference lists of the selected articles were manually searched for additional studies.

Main outcome(s): The upper airway volume and the minimum cross-section area analyzed by CВCT were selected as primary outcome.

Additional outcome(s): The airway space analyzed by cephalometric radiographs were selected as secondary outcome.

Quality assessment / Risk of bias analysis: For randomized clinical trials, the risk of bias tool of the Cochrane Collaboration was used to evaluate the risk of bias. The Methodological Index for Non-randomized Studies (MINORS) was used to evaluate the methodological quality of non-randomized clinical studies. The overall quality of evidence for outcome was assessed using the Grading of Recommendations Assessment, Development and Evaluation (GRADE) approach.

Strategy of data synthesis: The electronic search of Embase, Cochrane Library, and Medline (via PubMed) databases. Manual screening of three major orthodontic journals (European Journal of Orthodontics, American Journal of Orthodontics and Dentofacial Orthopedics, and Angle Orthodontist), and the reference lists of the selected articles were manually searched for additional studies.

Subgroup analysis: The subgroup analysis was performed to reduce the heterogeneity from different patient age.

Sensibility analysis: Sensitivity analysis was conducted to test the stability of some of the debatable results in the meta-analysis.

Country(ies) involved: China.

Keywords: Orthodontic, Extraction, Airway. 